\title{
Neuroprotective Effects of 4-phenylbutyric Acid and Its Derivatives: Possible Therapeutics for Neurodegenerative Diseases
}

\author{
Seisuke Mimori ${ }^{1}$, Toru Hosoi ${ }^{2}$, Masayuki Kaneko ${ }^{3}$, Koichiro Ozawa ${ }^{2}$, Tetsuto Kanzaki ${ }^{4}$, Akinori Nishi ${ }^{5}$ and \\ Yasuyuki Nomura ${ }^{5}$ \\ 1. Department of Clinical Medicine, Chiba Institute of Science, Choshi, 288-0025, Japan \\ 2. Department of Pharmacotherapy, Graduate School of Biomedical and Health Sciences, Hiroshima University, Hiroshima, \\ 734-8553, Japan \\ 3. Department of Biochemistry, Institute of Biomedical \& Health Sciences, Hiroshima University, Hiroshima, 734-8553, Japan \\ 4. Department of Drug Informatics, Graduate School and Faculty of Pharmaceutical Sciences, Chiba University, Chiba, 260-8675, \\ Japan \\ 5. Department of Pharmacology, Kurume University School of Medicine, Kurume, 830-0011, Japan
}

\begin{abstract}
The pathogenesis of neurodegenerative diseases such as Alzheimer's and Parkinson's diseases involves the aggregation of denatured and misfolded nascent proteins. Consequently, many pharmacological approaches have been developed to prevent protein aggregation. 4-Phenylbutyric acid (4-PBA) is a chemical chaperone that shows potential as a candidate drug for the treatment of neurodegenerative diseases. The main actions of chemical chaperones are the amelioration of unfolded proteins and the suppression of their aggregation, which result in protective effects against endoplasmic reticulum stress-induced neuronal cell death. Furthermore, 4-PBA exhibits inhibitory activity against histone deacetylases (HDACs). However, owing to the problematically high doses of 4-PBA currently required for therapeutic efficacy, the optimization of 4-PBA is crucial for its effective medicinal application. In the present review, we summarize the recent advances in research on the basic actions of 4-PBA and its derivatives. We also discuss whether these compounds could be viable therapeutic agents against neurodegenerative diseases.
\end{abstract}

Key words: 4-Phenylbutyric acid (4-PBA), neurodegenerative diseases, chemical chaperone, histone deacetylase (HDAC).

\section{Introduction}

The accumulation of aggregated proteins is a common feature in the pathogenesis of neurodegenerative diseases. Mutated genes code different amino acids, resulting in protein structures different from those of normal proteins. In general, a protein needs to be folded to work properly. Unfolded proteins are folded through the action of molecular chaperones such as heat shock proteins (HSPs). If a protein is not formed properly, the ubiquitin-proteasome system induces its selective degradation with the $26 \mathrm{~S}$ proteasome using

Corresponding author: Yasuyuki Nomura, Ph.D., research field: Cellular/Molecular Brain Pharmacology. mitochondrial ATP, which is vital for selective degradation, and thus for the survival of cells.

Disruption of the protein degradation system causes stagnation of ubiquitinated proteins, which results in the accumulation of aggregated unfolded proteins. These insoluble proteins constitute inclusion bodies in neurodegenerative diseases. Thus, abnormal proteins are thought to be involved in the pathogenesis of neurodegenerative diseases (Fig. 1). From a therapeutic point of view, the prevention of protein aggregation is critical, and recent studies have shown that anti-aggregation molecules may help to prevent aggregation of neurodegenerative diseases-related proteins. 


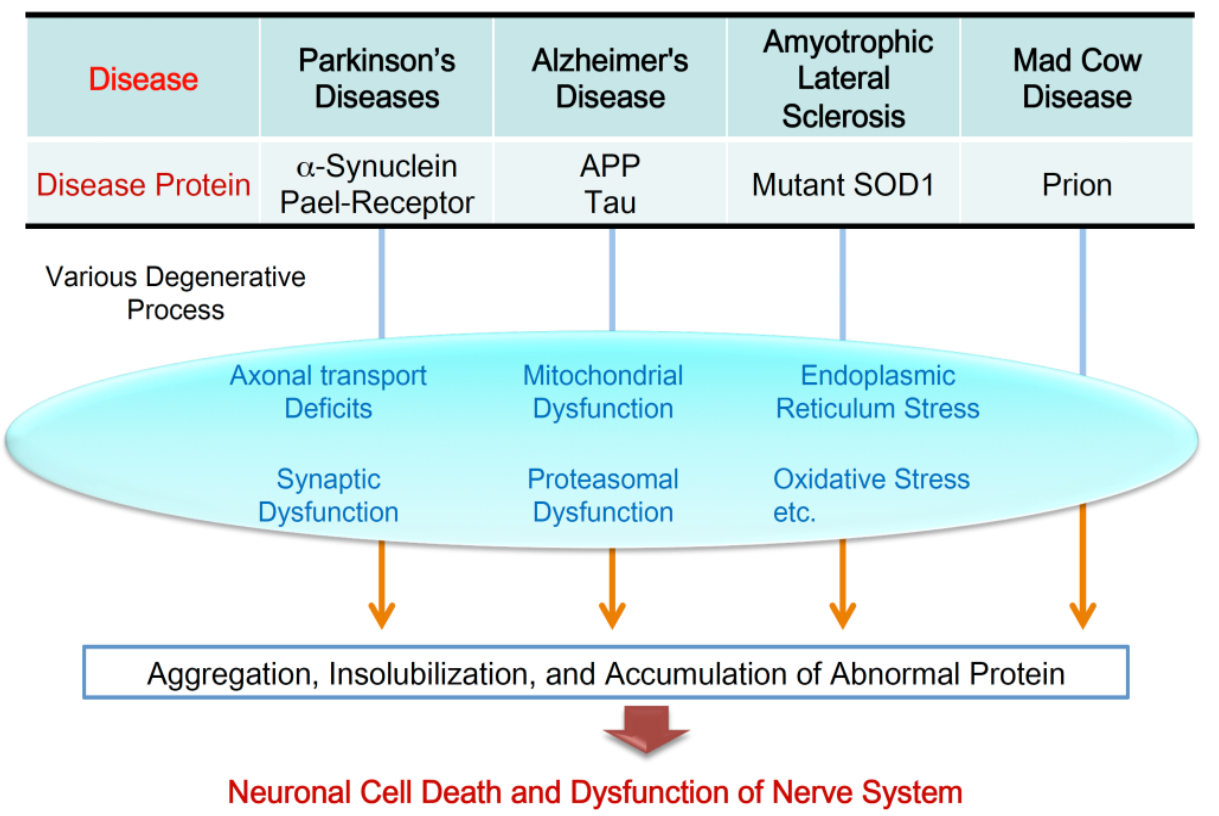

Fig. 1 Molecular mechanisms of neurodegenerative disease.

4-Phenylbutyric acid (4-PBA) is a small-molecular-weight fatty acid with a terminal aromatic group, and its sodium salt (sodium 4-phenyl butyrate) has been used for the treatment of urea cycle disorders [1]. This molecule is also used for the treatment of sickle cell diseases and thalassemia owing to its ability to activate $\beta$-globin transcription [2]. Moreover, 4-PBA is employed as an orally bioavailable agent for the treatment of spinal muscular atrophy (SMA) [3] and tumors [4]. Recently, the use of 4-PBA as a novel therapeutic agent for type 2 diabetes has been reported [5], and it has also been used as a treatment for familial hypercholesterolemia [6].

4-PBA has two main modes of action: as a chemical chaperone, and as a histone deacetylase (HDAC) inhibitor. Many studies have been published on the effects of 4-PBA in various biological models, and recently, the therapeutic effects of 4-PBA have been thoroughly classified and reviewed by Kolb et al. [7].

\section{4-PBA as a Chemical Chaperone}

Molecular chaperones such as HSPs provide cellular protection by preventing nascent proteins from misfolding, and reducing protein-protein aggregation. For example, Hsp70 is an intracellular chaperone that affects the amounts of misfolded and aggregated $\alpha$-synuclein ( $\alpha$-syn) both in vivo and in vitro [8]. The aggregation of nascent or denatured proteins is considered to be a major factor in the pathogenesis of neurodegenerative diseases, as this aggregation can induce endoplasmic reticulum (ER) stress. For further details, the reader is directed to Hosoi et al. (2015), who thoroughly reviewed the relationship between Alzheimer's disease (AD) and endoplasmic reticulum stress [9].

Chemical chaperones mimic the functions of intracellular molecular chaperones. 4-PBA appears to stabilize nascent proteins and HSPs, which are known to interact with mutated or unfolded proteins (Fig. 2). 4-PBA has been reported to restore mutated cystic fibrosis transmembrane conductance regulator (CFTR), a phenomenon that may be exploited for therapeutic effect [10-13]. Furthermore, mutant $\alpha_{1}$-antitrypsin ( $\alpha 1$-ATZ), which is retained in the ER of liver cells rather than being secreted into the blood and body fluids [14], can be folded by 4-PBA and may consequently be secreted. Moreover, 4-PBA exhibits 


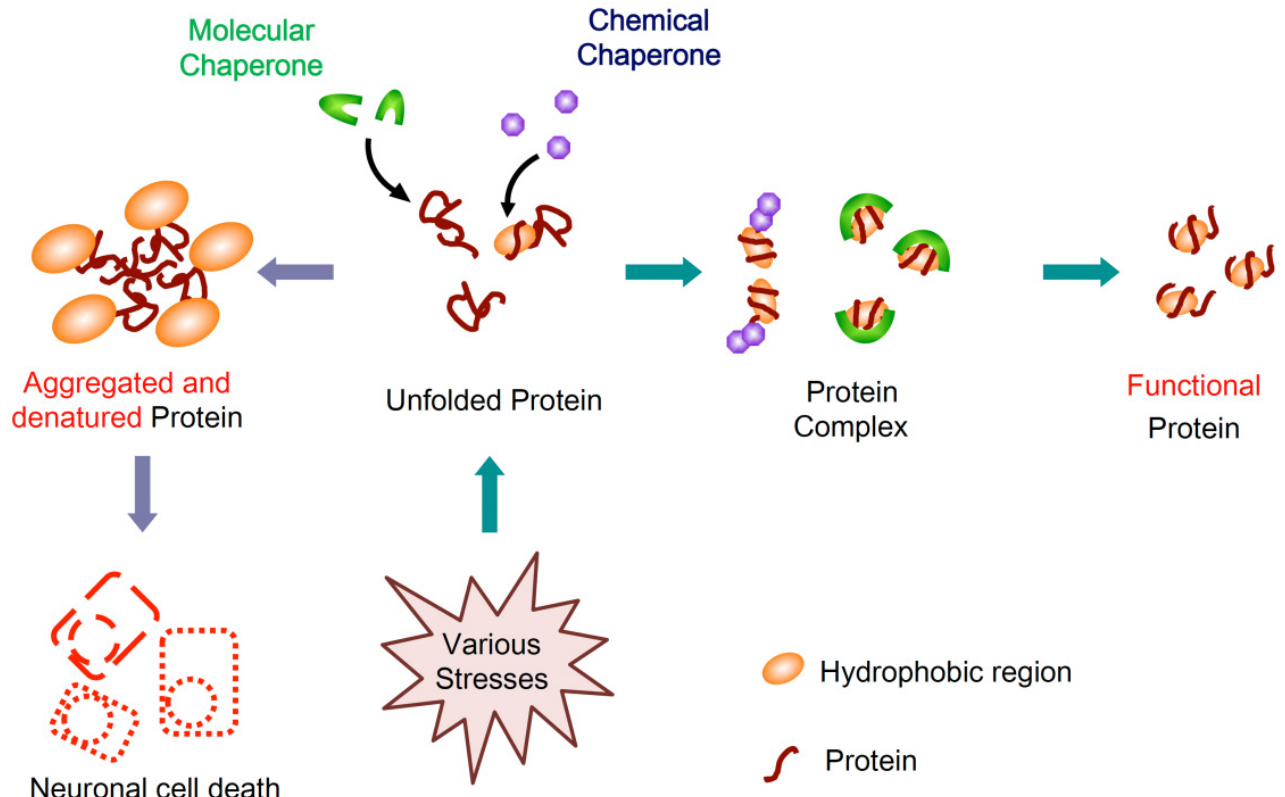

Fig. 2 4-PBA working as a molecular chaperone.

chemical chaperone activity, and has been reported to prevent the aggregation of denatured $\alpha$-lactalbumin and bovine serum albumin (BSA) in vitro [15]. 4-PBA has also been reported to restore normal expression levels of Parkin-associated endothelin receptor-like receptor (Pael-R), which is one of the genes responsible for Parkinson's disease (PD), and to suppress Pael-R-induced ER stress [16-18]. Thus, 4-PBA is an effective compound against a wide variety of aggregated-protein conditions. Furthermore, 4-PBA ameliorates palmitate-induced inhibition of glucose-stimulated insulin secretion (GSIS) [19], and has been reported to improve cataracts caused by mutant $\gamma$ D-crystallin in cultured cells [20].

\section{4-PBA as an HDAC Inhibitor}

In general, histone acetyltransferases (HATs) and HDACs catalyze the acetylation and deacetylation, respectively, of histones. Epigenetic gene expression is regulated by the balance of HATs and HDACs, and by the methylation of DNA. The acetylation of histones promotes their partial loosening, so that transcription factors can easily bind to the gene. This induces epigenetic transcription (which regulates cellular homeostasis) and the expression of a variety of genes. In many types of cancer, related proteins are constitutively overexpressed, and HDACs are thought to remediate this aberrant gene expression to its correct state. Thus, HDAC inhibitors have been developed as anticancer agents. These studies have already been summarized in several other excellent reviews [21-23].

Unlike cancer, neurodegenerative conditions are accompanied by collapsed homeostasis and declined turnover of proteins. However, both cancer and neurodegenerative diseases are accompanied by abnormal gene expression. In general, neurodegenerative diseases are presumed to be caused, or at least exacerbated, by the low acetylation of histones. Thus, HDAC inhibitors have recently become thought of as candidate therapeutic agents to improve the balance of gene expression, as reviewed by Das et al. (2016) [24].

Eighteen HDACs have been identified and classified into four categories by their homology, as reviewed by Zhang et al. (2015) [25]. Crystal structure analysis of several types of HDACs has been performed to facilitate the discovery of HDAC 
inhibitors [26-28]. To function as a catalytic enzyme, almost all HDACs require a metal ion, except for class III HDACs. 4-PBA is thought to chelate zinc ions, forming a complex that subsequently acts as an HDAC inhibitor. Butyrate also acts via the same mechanism as 4-PBA owing to its similar structure, as short chain fatty acids are involved in both [29]. Butyrate inhibits many HDACs, except for HDAC6, HDAC10, and class III HDACs. Furthermore, Valproic acid, a similar compound, is thought to enhance the anticonvulsant activity of GABA in suppressive synapses [30, 31].

HDACs have been reported to act upon the central nervous system in disorder models, and the inhibitory effects of HDACs are likely to play an important role in the treatment of mood disorders. For example, 4-PBA is reported to ameliorate cognitive deficit and reduction of tau pathologies in an $\mathrm{AD}$ mouse model [32]. Moreover, systemic administration of 4-PBA has been reported to reinstate fear learning in the $\mathrm{Tg} 2576$ AD mouse model [33], and 4-PBA has been shown to exert significant neuroprotective effects in a PD mouse model [34-36]. It has also been reported that 4-PBA has a neuroprotective effect in the N171-82Q transgenic mouse model of Huntington's diseases (HD) [37]. Administration of 4-PBA increased brain histone acetylation and decreased histone methylation levels, as assessed by both immunocytochemistry and Western blots. Moreover, 4-PBA was shown to significantly extended survival and improve clinical and neuropathological phenotypes in G93A transgenic amyotrophic lateral sclerosis (ALS) mice [38], and the effectiveness of 4-PBA against SMA has been frequently reported $[3,39,40]$. Improvement of these conditions is suggested to be mediated by the inhibitory activity and/or chemical chaperone activity of HDAC inhibitors [3, 38-40].

\section{Development of 4-PBA in our Research}

4-PBA protects against cerebral ischemic injury and ER stress-induced neuronal death [41], which indicates its chemical chaperone activity. It also prevents the aggregation of reduced $\alpha$-lactalbumin with denatured BSA [15].

Unfortunately, 4-PBA is required in high doses to prevent protein aggregation. Consequently, in order to improve the efficiency of the chemical chaperone activity of 4-PBA, we investigated the structure-activity relationship (SAR) in 4-PBA analogs with different numbers of carbon atoms in the fatty acid chain (Fig. 3) [42]. The aggregation inhibitory effect in vitro increased relative to the number of carbons from three to six. The longer carbon chain molecules were effective against ER stress-induced cell death by tunicamycin in SH-SY5Y neuroblastoma cells, whereas 3-phenylpropanoic acid (3-PPA) and 4-PBA only protected against Pael-R-induced cell death. Moreover, localization of the overexpressed Pael-R was shifted from the ER to the cytoplasmic membrane by 3-PPA and 4-PBA.

As another strategy to optimize 4-PBA, we investigated synthetic methoxy-substituted 4-PBA derivatives (Fig. 4) [43]. 4-(4-Methoxyphenyl) butanoic acid (4-MPB) showed protective effects against ER stress-induced neuronal cell death. However, further optimization is necessary for clinical use, since the required dose of 4-MPB was not significantly smaller than that of 4-PBA.

It is important to determine whether it is the chemical chaperone or HDAC inhibitor activity of 4-PBA that reduces ER stress-induced cell death. We showed that 4-PBA protects against neuronal cell death by primarily acting as a chemical chaperone rather than as an HDAC inhibitor using three simple 4-( $p$-substituted phenyl) butyric acids [44]. 4-(4-Aminophenyl) butanoic acid (4-APB) exhibited high affinity an HDAC7/4-PBA-derivative binding model. In fact, 4-APB showed a stronger HDAC inhibitory effect than the other derivatives. However, 4-APB did not exhibit protective effects against ER stress-induced cell death or chemical chaperone activity in vitro, whereas 4-MPB exhibited slightly 
(A)

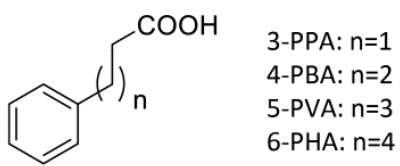

(B)

\begin{tabular}{|c|c|c|}
\hline $\begin{array}{l}\text { Chemical } \\
\text { chaperone } \\
\text { activity }\end{array}$ & $\begin{array}{l}\text { Protective effects } \\
\text { against cell death } \\
\text { (tunicamycin) }\end{array}$ & $\begin{array}{l}\text { Protective effects } \\
\text { against cell death } \\
\text { (Pael-R overexpression) }\end{array}$ \\
\hline
\end{tabular}

\begin{tabular}{ccccc}
\hline 3-РРА & + & + & ++++ & +++ \\
\hline 4-РВА & ++ & ++ & ++++ & ++++ \\
\hline 5-PVA & ++ & +++ & ++ & ++ \\
\hline 6-РНA & ++++ & ++++ & ++ & +
\end{tabular}

Fig. 3 Activity comparison of 4-PBA derivatives with different numbers of carbons in the fatty acid chain. (A) 4-PBA derivatives. (B) The results of our study (Ref. [42]). Relative efficacy is indicated by the number of plus (+) symbols.

(A)

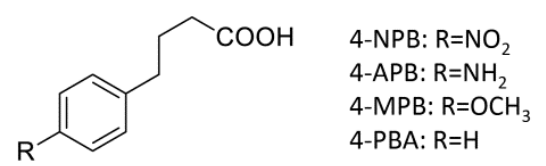

(B)

Chemical chaperone activity
Protective effects against cell death (tunicamycin)

\begin{tabular}{cccc} 
4-NPB & +++ & ++ & +++ \\
\hline 4-AРB & + & +++++ & + \\
\hline 4-MPB & ++++ & +++ & +++ \\
\hline 4-РBA & +++ & ++++ & +++
\end{tabular}

Fig. 4 Activity comparison of the 4-PBA derivatives replaced with the para position. (A) 4-PBA derivatives replaced with the para position. (B) The results of our study (Ref. [44]). Relative evaluation using the number of plus (+) symbols.

stronger chemical chaperone activity than that of 4-PBA. Conversely, 4-MPB exhibited weaker HDAC inhibitory activity than that of 4-PBA. These data suggest that the inhibitory effect of 4-PBA on cell death is due to its aggregation-inhibitory activity (i.e., in vitro chemical chaperon activity) (Fig. 4).

We are currently conducting drug discovery research focusing on the chemical chaperone activity of the benzene ring of 4-PBA, which can interact with other benzene rings through $\pi-\pi$ interactions (or 


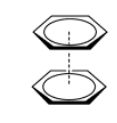

1

Stacking

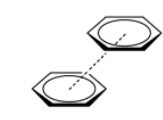

2

Offset stacking

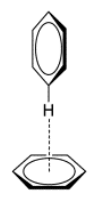

3

Edge-to-face aromatic interaction

Fig. 5 Possible interactions of benzene rings.

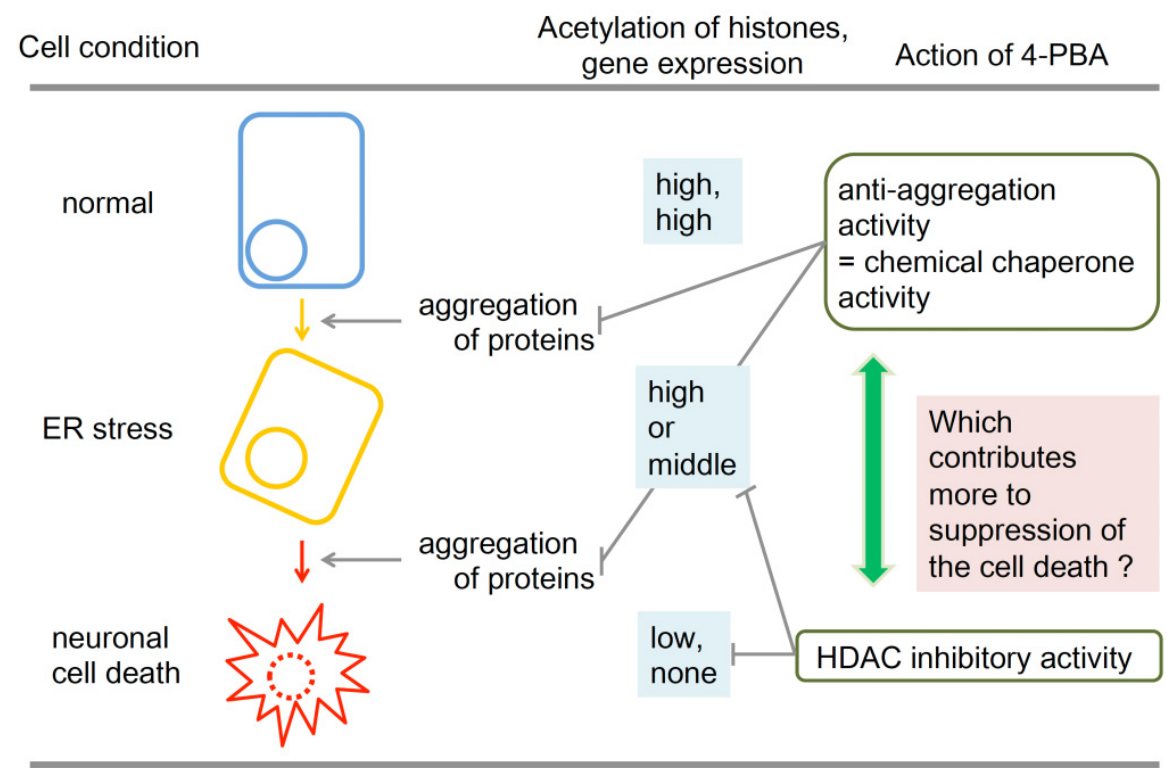

Fig. 6 Hypothetical mechanism of 4-PBA against cell death.

stacking). The possible interactions are shown in Fig. 5. The electron cloud area may be directly related to the anti-aggregation activity. This model appears to require a large amount of 4-PBA for the assembly of denatured proteins. We assume that the electron clouds of the benzene rings interact with the aggregates of denatured proteins. Thus, we attempted to expand the electron cloud area by synthesizing naphthalene-based 4-PBA analogs [45]. The chemical chaperone activity increased relative to that of 4-PBA. However, the synthesis of these analogs entailed the use of highly toxic reagents, so further work on 4-PBA analogs with different frameworks to naphthalene is underway in our group.

\section{Conclusions}

4-PBA is a biologically active small molecule that has already been demonstrated to be safe, and is approved by the FDA. However, further study is required in order to fully understand this interesting compound. We believe that the main effects of 4-PBA are its protein-aggregation inhibitory activity and its HDAC inhibitory activity (Fig. 6). Histones are thought to be over-deacetylated in neurodegenerative conditions; therefore, the control of deacetylation is a promising therapeutic strategy for their treatment.

\section{Acknowledgments}

We especially thank Dr. Ezio Giacobini. Moreover, we thank Drs. Y. Okuma, Y. Murakami, H. Hamana, H. Ohtaka and K. Kawada for their helpful suggestions in the preparation of this paper. The present study was supported by a Grant-in-Aid for Scientific Research from the Ministry of Education, Science, Sports and Culture, Japan. The authors would like to thank to Enago for the English language review. 


\section{Conflict of Interest}

The authors declare no conflict of interest.

\section{References}

[1] Brusilow, S. W., and Maestre, N. E. 1996. "Urea cycle disorders: diagonosis, pathophysiology, and therapy." Advances in Pediatrics 43: 127-70.

[2] Collins, A. F., Pearson, H. A., Giardina, P., McDonagh, K. T., Brusilow, S. W., and Dover, G. J. 1995. "Oral sodium phenylbutyrate therapy in homozygous beta thalassemia: a clinical trial." Blood 85 (1): 43-9.

[3] Mercuri, E., Bertini, E., Messina, S., Pelliccioni, M., D'Amico, A., Colitto, F., Mirabella, M., Tiziano, F. D., Vitali, T., Angelozzi, C., Kinali, M., Main, and M., Brahe, C. 2004. "Pilot trial of phenylbutyrate in spinal muscular atrophy." Neuromuscular Disorders 14 (2): 130-5.

[4] Carducci, M. A., Gilbert, J., Bowling, M. K., Noe, D., Eisenberger, M. A., Sinibaldi, V., Zabelina, Y., Chen, T. L., Grochow, L. B., and Donehower, R. C. 2001. "A phase I clinical and pharmacological evaluation of sodium phenylbutyrate on an 120-h infusion schedule." Clinical Cancer Research 7 (10): 3047-55.

[5] Ozcan, U., Yilmaz, E., Ozcan, L., Furuhashi, M., Vaillancourt, E., Smith, R.O., Görgün, C. Z., and Hotamisligil, G. S. 2006. "Chemical chaperones reduce ER stress and restore glucose homeostasis in a mouse model of type 2 diabetes." Science 313 (5790): 1137-40.

[6] Tevten, K., Holla, Ø. L., Ranheim, T., Berge, K. E., Leren, T. P., and Kulseth, M. A. 2007. "4-phenylbutyrate restores the functionality of a misfolded mutant low-density lipoprotein receptor." The FEBS Journal 274 (8): 1881-93.

[7] Kolb, P. S., Ayaub, E. A., Zhou, W., Yum, V., Dickhout, J. G., and Ask, K. 2015. "The therapeutic effects of 4-phenylbutyric acid in maintaining proteostasis." International Journal of Biochemistry and Cell Biology 61: 45-52.

[8] Klucken, J., Shin, Y., Hyman, B. T., and McLean, P. J. 2004. "A single amino acid substitution differentiates Hsp70-dependent effects on alpha-synuclein degradation and toxicity." Biochemical and Biophysical Research Communications 325 (1): 367-73.

[9] Hosoi, T., Nomura, J., Ozawa, K., Nishi, A., and Nomura, Y. 2015. "Possible involvement of endoplasmic reticulum stress in the pathogenesis of Alzheimer's disease." Endoplasmic Reticulum Stress in Diseases 2: 107-18.

[10] Rubenstein, R. C., Egan, M, E., and Zeitlin, P. L. 1997. "In vitro pharmacologic restoration of CFTR-mediated chloride transport with sodium 4-phenylbutyrate in cystic fibrosis epithelial cells containing delta F508-CFTR." Journal of Clinical Investigation 100 (10): 2457-65.
[11] Rubenstein, R. C., and Zeitlin, P. L. 1998. "A pilot clinical trial of oral sodium 4-phenylbutyrate (Buphenyl) in deltaF508-homozygous cystic fibrosis patients: partial restoration of nasal epithelial CFTR function." American Journal of Respiratory and Critical Care Medicine 157 (2): 484-90.

[12] Zeitlin, P. L., Diener-West, M., Rubenstein, R. C., Boyle, M. P., Lee, C. K., and Brass-Emst, L., 2002. "Evidence of CFTR in cystic fibrosis after systemic administration of 4-phenylbutyrate." Molecular Therapy 6 (1): 119-26.

[13] Powell, K., and Zeitlin, P. L. 2002. "Therapeutic approaches to repair defects in deltaF508 CGTR folding and cellular targeting." Advanced Drug Delivery Reviews 54 (11): 1395-408.

[14] Burrows, J. A., Willis, L. K., and Perlmutter, D. H. 2000. "Chemical chaperones mediate increased seretion of mutant alpha 1-antitrypsin (alpha 1-AT) Z: A potential pharmacological strategy for prevention of liver injury and emphysema in alpha 1-AT deficiency." Proceedings of the National Academy of Sciences of the United States of America 97 (4): 838-43.

[15] Kubota, K., Niimura, Y., Kaneko, M., Okuma, Y., Sugai, M., Omura, T., Uesugi, M., Uehara, T., Hosoi, T., and Nomura, Y. 2006. "Suppressive effects of 4-phenylbutyrate on the aggregation of Pael receptors and endoplasmic reticulum stress." Journal of Neurochemistry 97 (5): 1259-68.

[16] Imai, Y., Soda, M., and Takahashi, R. 2000. "Parkin suppresses unfolded protein stress-induced cell death through its E3 ubiquitin-protein ligase activity." Journal of Biological Chemistry 275 (46): 35661-4.

[17] Imai, Y., Soda, M., Inoue, H., Hattori, N., Mizuno, Y., and Takahashi, R. 2001. "An unfolded putative transmembrane polypeptide, which can lead to endoplasmic reticulum stress, is a substrate of Parkin." Cell 105 (7): 891-902.

[18] Yang, Y., Nishimura, I., Imai, Y., Takahashi, R., and Lu, B. 2003. "Parkin suppresses dopaminergic neuron-selective neurotoxicity induced by Pael-R in Drosophila." Neuron 37(6): 911-24.

[19] Choi, S. E., Lee, Y. J., Jang, H. J., Lee, K. W., Kim, Y. S., Jun, H. S., Kang S. S., Chun, J., and Kang, Y. 2008. “A chemical chaperone 4-PBA ameliorates palmitate-induced inhibition of glucose-stimulated insulin secretion (GSIS)." Archives of Biochemistry and Biophysics 475 (2): 109-14.

[20] Gong, B., Zhang, L. Y., Lam, D. S., Pang, C. P., and Yam, G. H. 2010. "Sodium 4-phenylbutyrate ameliorates the effects of cataract-causing mutant gammaD-crystallin in cultured cells." Molecular Vision 16: 997-1003.

[21] Parbin, S., Kar, S., Shilpi, A., Sengupta, D., Deb, M., Rath, S. K., and Patra, S. K. 2014. "Histone deacetylases: 
a saga of perturbed acetylation homeostasis in cancer." Journal of Histochemistry and Cytochemistry, 62 (1): 11-33.

[22] Cruz, F. D., and Matushansky, I. 2012. "Solid tumor differentiation therapy-is it possible?" Oncotarget 3 (5): 559-67.

[23] Dokmanovic, M., Clarke, C., and Marks, P. A. 2007. "Histone deacetylase inhibitors: overview and perspectives." Molecular Cancer Research 5 (10): 981-9.

[24] Das, Gupta. K., Shakespear, M. R., Iyer, A., Fairlie, D. P., and Sweet, M. J. 2016. "Histone deacetylases in monocyte/macrophage development, activation and metabolism: refining HDAC targets for inflammatory and infectious diseases." Clinical and Translational Immunology 5 (1): e62.

[25] Zhang, J., and Zhong, Q. 2014. "Histone deacetylase inhibitors and cell death." Cellular and Molecular Life Sciences 71 (20): 3885-901.

[26] Schäfer, S., Saunders, L., Eliseeva, E., Velena, A., Jung, M., Schwienhorst, A., Strasser, A., Dickmanns, A., Ficner, R., Schlimme, S., Sippl, W., Verdin, E., and Jung, M. 2008. "Phenylalanine-containing hydroxamic acids as selective inhibitors of class IIb histone deacetylases (HDACs)." Bioorganic and Medical Chemistry 16 (4): 2011-33.

[27] Bürli, R. W., Luckhurst, C. A., Aziz, O., Matthews, K. L., Yates, D., Lyons, K. A., Beconi, M., McAllister, G., Breccia, P., Stott, A. J., Penrose, S. D., Wall, M., Lamers, M., Leonard, P., Müller, I., Richardson, C. M., Jarvis, R., Stones, L., Hughes, S., Wishart, G., Haughan, A. F., O’Connell, C., Mead, T., McNeil, H., Vann, J., Mangette, J., Maillard, M., Beaumont, V., Munoz-Sanjuan, I., and Dominguez, C. 2013. "Design, synthesis, and biological evaluation of potent and selective class IIa histone deacetylase (HDAC) inhibitors as a potential therapy for Huntington's disease." Journal of Medicinal Chemistry 56 (24): 9934-54.

[28] Whitehead, L., Dobler, M. R., Radetich, B., Zhu, Y., Atadja, P. W., Claiborne, T., Grob, J.E., McRiner, A., Pancost, M. R., Patnaik, A., Shao, W., Shultz, M., Tichkule, R., Tommasi, R. A., Vash, B., Wang, P., and Stams, T. 2011. "Human HDAC isoform selectivity achieved via exploitation of the acetate release channel with structurally unique small molecule inhibitors." Bioorganic and Medicinal Chemistry 19 (15): 4626-34.

[29] Vidali, G., Boffa, L. C., Bradbury, E. M., and Allfrey, V. G. 1978. "Butyrate suppression of histone deacetylation leads to accumulation of multiacetylated forms of histones $\mathrm{H} 3$ and $\mathrm{H} 4$ and increased DNase I sensitivity of the associated DNA sequences." Proceedings of the National Academy of Sciences of the United States of America 75 (7): 2239-43.
[30] Phiel, C. J., Zhang, F., Huang, E. Y., Guenther, M. G., Lazar, M. A., and Klein, P. S. 2001. "Histone deacetylase is a direct target of valproic acid, a potent anticonvulsant, mood stabilizer, and teratogen." Journal of Biological Chemistry 276 (39): 36734-41.

[31] Göttlicher, M., Minucci, S., Zhu, P., Krämer, O. H., Schimpf, A., Giavara, S., Sleeman, J. P., Lo Coco, F., Nervi, C., Pelicci, P. G., and Heinzel, T. 2001. "Valproic acid defines a novel class of HDAC inhibitors inducing differentiation of transformed cells." EMBO Journal 20 (24): 6969-78.

[32] Ricobaraza, A., Cuadrado-Tejedor, M., Pérez-Mediavilla, A., Frechilla, D., Del Río, J., and García-Osta, A. 2009. "Phenylbutyrate ameliorates cognitive deficit and reduces tau pathology in an Alzheimer's disease mouse model." Neuropsychopharmacology 34 (7): 1721-32.

[33] Ricobaraza, A., Cuadrado-Tejedor, M., Marco, S., Pérez-Otaño, I., and García-Osta, A. 2010. "Phenylbutyrate rescues dendritic spine loss associated with memory deficits in a mouse model of Alzheimer disease." Hippocampus 22 (5): 1040-50.

[34] Inden, M., Kitamura, Y., Takeuchi, H., Yanagida, T., Takata, K., Kobayashi, Y., Taniguchi, T., Yoshimoto, K., Kaneko, M., Okuma, Y., Taira, T., Ariga, H., and Shimohama, S. 2007. "Neurodegeneration of mouse nigrostriatal dopaminergic system induced by repeated oral administration of rotenone is prevented by 4-phenylbutyrate, a chemical chaperone." Journal of Neurochemistry 101 (6): 1491-504.

[35] Ono, K., Ikemoto, M., Kawarabayashi, T., Ikeda, M., Nishinakagawa, T., Hosokawa, M., Shoji, M., Takahashi, M., and Nakashima, M. 2009. "A chemical chaperone, sodium 4-phenylbutyric acid, attenuates the pathogenic potency in human alpha-synuclein A30P + A53T transgenic mice." Parkinsonism and Related Disorders 15 (9): 649-5.

[36] Gardian, G., Yang, L., Cleren, C., Calingasan, N. Y., Klivenyi, P., and Beal, M. F. 2004. "Neuroprotective effects of phenylbutyrate against MPTP neurotoxicity." Neuromolecular Medicine 5 (3): 235-41.

[37] Gardian, G., Browne, S. E., Choi, D. K., Klivenyi, P., Gregorio, J., Kubilus, J. K., Ryu, H., Langley, B., Ratan, R. R., Ferrante, R. J., and Beal, M. F. 2005. "Neuroprotective effects of phenylbutyrate in the N171-82Q transgenic mouse model of Huntington's disease." Journal of Biological Chemistry 280 (1): 556-63.

[38] Ryu, H., Smith, K., Camelo, S. I., Carreras, I., Lee, J., Iglesias, A. H., Dangond, F., Cormier, K. A., Cudkowicz, M. E., Brown, R. H. Jr., and Ferrante, R. J. 2005. "Sodium phenylbutyrate prolongs survival and regulates expression of anti-apoptotic genes in transgenic 
amyotrophic lateral sclerosis mice." Journal of Neurochemistry 93 (5): 1087-98.

[39] Andreassi, C., Angelozzi, C., Tiziano, F. D., Vitali, T., De Vincenzi, E., Boninsegna, A., Villanova, M., Bertini, E., Pini, A., Neri, G., and Brahe, C. 2004. "Phenylbutyrate increases SMN expression in vitro: relevance for treatment of spinal muscular atrophy.” European Journal of Human Genetics 12 (1): 59-65.

[40] Brahe, C., Vitali, T., Tiziano, F. D., Angelozzi, C., Pinto, A. M., Borgo, F., Moscato, U., Bertini, E., Mercuri, E., and Neri, G. 2005. "Phenylbutyrate increases SMN gene expression in spinal muscular atrophy patients." European Journal of Human Genetics 13 (2): 256-9.

[41] Qi, X., Hosoi, T., Okuma, Y., Kaneko, M., and Nomura, Y. 2004. "Sodium 4-phenylbutyrate protects against cerebral ischemic injury." Molecular Pharmacology 66 (4): 899-908.

[42] Mimori, S., Okuma, Y., Kaneko, M., Kawada, K., Hosoi, T., Ozawa, K., Nomura, Y., and Hamana, H. 2012. "Protective effects of 4-phenylbutyrate derivatives on the neuronal cell death and endoplasmic reticulum stress." Biological and Pharmaceutical Bulletin 35 (1): 84-90.

[43] Mimori, S., Okuma, Y., Kaneko, M., Kawada, K., Nomura, Y, Murakami, Y., and Hamana, H. 2013. "Discovery of synthetic methoxy-substituted 4-phenylbutyric acid derivatives as chemical chaperone." Chemistry Letters 42: 1051-2.

[44] Mimori, S., Ohtaka, H., Koshikawa, Y., Kawada, K., Kaneko, M., Okuma, Y., Nomura, Y., Murakami, Y., and Hamana, H. 2013. "4-Phenylbutyric acid protects against neuronal cell death by primarily acting as a chemical chaperone rather than histone deacetylase inhibitor." Bioorganic and Medical Chemistry Letters 23 (21): 6015-8.

[45] Mimori, S., Koshikawa, Y., Mashima, Y., Mitsunaga, K., Kawada, K., Kaneko, M., Okuma, Y., Nomura, Y., Murakami, Y., Kanzaki, T., and Hamana, H. 2015. "Evaluation of synthetic naphthalene derivatives as novel chemical chaperones that mimic 4-phenylbutyric acid." Bioorganic and Medical Chemistry Letters 25 (4): 811-4. 